\title{
Telegram como herramienta para periodistas: Percepción y uso
}

\section{Telegram as a tool for journalists: Perception and use}

\author{
Sánchez Gonzales, H. M. y Martos Moreno, J. ${ }^{1}$ \\ Recibido: 15-05-2020 - Aceptado: 20-08-2020 \\ https://doi.org/10.26441/RC19.2-2020-A14
}

RESUMEN: La búsqueda de modelos de negocio alternativos y el auge de las nuevas tecnologías han propiciado una transformación en las rutinas de los periodistas y en el rol de las audiencias. Este debate se ha acrecentado a raíz del protagonismo de plataformas como las redes sociales o las aplicaciones de mensajería instantánea. El presente estudio analiza la instrumentalización de Telegram en las rutinas periodísticas debido a la utilidad, seguridad y privacidad que ofrece la plataforma. Gracias a estas cualidades técnicas que presenta, Telegram ha irrumpido en las prácticas rutinarias de los periodistas, especialmente, como fuente de información personalizada y como canal de distribución de noticias. La percepción de los profesionales y estudiantes de comunicación es positiva, en relación al consumo de información a través de las breaking news y titulares relevantes por la sencillez y originalidad de uso de la aplicación. Sin embargo, pese a su incursión en la actividad periodística, su escasa implantación social todavía representa un importante escollo para su desarrollo a nivel de la población.

Palabras clave: Telegram; periodismo; rutinas profesionales; periodista; audiencia; nuevas tecnologías.

\begin{abstract}
The search for alternative business models and the rise of new technologies have led to a transformation in the routines of journalists and the role of audiences. This debate has increased as a result of the prominence of platforms such as social networks or instant messaging applications. This study analyzes the implementation of Telegram in journalistic routines due to the usefulness, security and privacy offered by the platform. Thanks to these technical qualities, Telegram has broken into the routine practices of journalists, especially as a source of personalized information and as a news distribution channel. The perception of communication professionals and students is positive, in relation to the consumption of information through breaking news and relevant headlines due to the simplicity and originality of use of the application. However, in spite of its incursion into journalistic activity, its scarce social implantation still represents an important obstacle to its development at the level of the population.
\end{abstract}

Keywords: Telegram; journalism; professional routines; journalism; audience; new technologies.

\footnotetext{
${ }^{1}$ Hada M. Sánchez Gonzales es Doctora en Periodismo, profesora Titular de Universidad, directora del Laboratorio de Proyectos en Comunicación y Vicedecana de Comunicación y Relaciones Institucionales de la Facultad de Comunicación de la Universidad de Sevilla. Cuenta con un sexenio de investigación y forma parte del cuerpo de evaluadores de la ANEP. misago@us.es, https://orcid.org/0000-0001-5078-2184

Javier Martos Moreno es Periodista y Máster en Comunicación Institucional y Política en la Universidad de Sevilla. Doctorando Interuniversitario en Comunicación de la Universidad de Sevilla. Ha desempeñado labores de redacción en medios de comunicación y en gabinetes de prensa. javiermm94@gmail.com, https://orcid.org/00000002-1659-3723
} 


\section{Introducción}

Los cambios tecnológicos han traído consigo posibilidades comunicativas en el periodismo. Los procesos de digitalización han alterado los cimientos de las empresas periodísticas y de las rutinas profesionales de la comunicación. Sin embargo, la transformación digital no debe asumirse como una tecnología avanzada o de vanguardia, sino, como una estrategia que considera el aspecto cultural, organizativo y de conocimiento, además del tecnológico (Kane, 2017; Álvarez, Capelo y Álvarez, 2019). Esto permite a las industrias periodísticas hacer frente al entorno competitivo y conservar a la audiencia, teniendo en cuenta, la innovación como eje vertebral.

La innovación periodística (Sánchez, 2005) es un factor clave en la madurez digital y transformación de las redacciones frente a la crisis de cambio en los modelos de negocio. Se convierte en un componente clave para la transmisión del mensaje al usuario porque transforma los sistemas culturales tradicionales de forma exponencial sin dejar de lado el valor axiológico (Echeverría, 2000) y sociocultural. De ahí que muchos medios de comunicación hayan incorporado los labs, laboratorios de innovación, como una unidad más de la empresa periodística que opera con autonomía.

Ante la búsqueda de nuevos modelos de negocio y protagonismo de las nuevas tecnologías, autores y académicos (Casasús, 2001; Súarez-Villegas, 2015) llaman a la reflexión acerca del papel del periodismo en la era digital. Otros (Micó, Canavilhas, Masip, Ruiz; 2008) abordan este debate desde el punto de vista de la deontología de la profesión. Los periodistas deben reflexionar sobre la tecnología utilizada para informar y cómo repercute en el proceso de búsqueda de la verdad.

Con la popularización de los medios digitales, especialmente redes sociales y plataformas de mensajería, se han disparado los interrogantes acerca de su uso en la profesión periodística. Frente a la incorporación de estas vías de comunicación en las rutinas de medios y periodistas, surgen corrientes que apelan a los principios éticos del periodismo (Casasús, 2001) y a su aplicación a los nuevos formatos digitales (Herrera-Damas, 2013).

Actualmente, asistimos a un periodismo continuo, personalizado y participativo con capacidad multimedia que adquiere su identidad en la Red. De acuerdo con la última actualización del consorcio World Wide Web (W3C), es posible que estemos frente a una generación que propicia una mayor interacción en tiempo real, el lenguaje de los sentidos y de las emociones con facilidad (Sánchez, 2018). Se trata de una nueva forma cultural y social de comunicación que requiere nuevos planteamientos en la labor del periodista.

En este contexto, la comunicación líquida (Bauman, 2007) cobra protagonismo y una de sus principales manifestaciones son las redes sociales, ancladas en el ecosistema social media (Kaplan; Haenlein, 2010; Sánchez y García, 2010). Éstas son definidas como la "miscelánea de actores, sujetos, grupos y comunidades de nodos que han conexionado a través de un conjunto de relaciones en base a intereses comunes dentro de un espacio de convivencia” (Sánchez y García, 2010: 373).

El protagonismo creciente de las redes sociales ha despertado un amplio debate sobre su uso en la profesión periodística, debido a ciertas prácticas reprobables como el uso abusivo de noticias de agencia, la emisión de imágenes éticamente discutibles (Micó et al, 2008) o la publicación de noticias falsas o manipuladas (Shu, Sliva, Wang, Tang, Liu, 2017). Todo ello ha desembocado en la existencia de una profunda percepción de crisis de credibilidad instalada en la profesión que se traduce en la pérdida de confianza por parte de los ciudadanos.

A pesar de esto, las redes se han convertido en parte de las rutinas periodísticas de cualquier medio de comunicación (Herrero Curiel, 2015) en cuanto a las tareas de transmisión y obtención de información, contribuyendo además al impulso de la participación de los ciudadanos (Masip, Guallar, Suau, Ruiz-Caballero, Peralta, 2015) y a las mejoras en cuanto a la inmediatez e interactividad (Meso, Agirreazkuenaga, Larrondo, 2015). 
También, han surgido otras plataformas provocadas por la irrupción y consumo de los dispositivos móviles en España (Varona, Sánchez y Arrocha, 2017): las aplicaciones de mensajería instantánea. A diferencia de las redes sociales, éstas permiten distribuir la información de forma personalizada a la audiencia en entornos privados. Se trata de WhatsApp, Snapchat o Telegram. En concreto, esta última se ha convertido en una de las plataformas preferidas por el público para comunicarse (Sánchez y Martos, 2018) y resulta especialmente interesante para distribuir noticias por la influencia que pueden alcanzar estos contenidos.

Por ello, en este estudio nos centraremos en Telegram, servicio de mensajería creado en 2013. A pesar de no ser consumido por los usuarios de igual forma que WhatsApp o Facebook Messenger en España, aspira a ser una de las aplicaciones preferidas de los medios de comunicación por su utilidad. Esto también repercute en las competencias de los profesionales porque entra dentro de las rutinas periodísticas. Ha logrado asentarse en el periodismo (García, 2016) gracias a su carácter inmediato y, especialmente, por la seguridad y privacidad que ofrece (Martín Granados, 2016).

Los hábitos de consumo de la audiencia han cambiado, así como su papel activo en el sector periodístico. El usuario, convertido hoy en "prosumidor" (Toffler, 1980), "no sólo consume información, sino que interactúa y produce" (Sánchez, 2016: 68). Se han abierto nuevas vías para la producción (Hermida y Thurman, 2008), el consumo (Casero, 2012) y la distribución de contenidos.

El poder simbólico de la organización cultural y la creación de la realidad social (Thompson, 1985) ha dejado de ser monopolio exclusivo de los medios debido a la irrupción de las nuevas plataformas digitales y las audiencias activas. El periodista ya no dispone en exclusiva del acceso a las fuentes y ha perdido la hegemonía de la información ante el nuevo rol de la audiencia (Micó et al, 2008).

Ante este cambio de roles en la sociedad digital, el profesional de la información debe diferenciarse por su compromiso ético, la búsqueda de la verdad (Casasús, 2001) y por un sentido de la responsabilidad (Singer, 2006) que permita desarrollar su rol social, fundamentado en la confianza (Micó et al, 2008).

Otros autores (Breese y Luengo, 2016) han evaluado el impacto de este cambio de prácticas periodísticas como fuente de "crisis" para el periodismo. Este tipo de innovaciones producen cambios económicos y desafíos en las dinámicas profesionales que, en muchos casos, obligan a los periodistas a producir y distribuir noticias que evitan la investigación en profundidad por las actualizaciones constantes.

La caída en los ingresos publicitarios de los medios de comunicación tradicionales y la pérdida de confianza de la audiencia se suman a esta crisis. Empero, el modelo de negocio de los contenidos digitales está cambiando. En las redacciones se empezará hablar del término "funnel o embudo de conversión", estrategia que se dirige a la búsqueda de fidelidad con la audiencia y no a la captación de esta, como se venía haciendo hasta ahora. El objetivo sería lograr "la suscripción, el pago y la recomendación". Igualmente, cobra relevancia el desarrollo de productos, contenidos y funcionalidades que potencien un compromiso con el usuario (Muñoz, 2019).

Este nuevo escenario nos lleva hablar de nuevas competencias profesionales, nuevas rutinas profesionales por parte de los periodistas (Pérez y Micó 2015) y nuevas prácticas en el periodismo. Las empresas demandan periodistas adaptados a las tecnologías y a las necesidades de la audiencia teniendo en cuenta "la esencia del periodismo en estado puro" (Sánchez, 2016: 79). Estos deben contar con dos aspectos centrales (López, Rodriguez y Pereira, 2017): los elementos básicos del periodismo y con la dimensión tecnológica. Deben entender, adaptarse y disponer de conocimientos para el trabajo individual o en equipo.

Sin embargo, algunos periodistas han "manifestado resistencia, negándose firmemente a subordinar su ética profesional a lo que ellos ven como la lógica profana y contaminante del mercado y la 
tecnología" (Alexander, Breese y Luengo, 2016). También Erdal (2007) alega que la inclusión de nuevos formatos digitales en las redacciones causa altos niveles de angustia debido al cambio constante de prácticas profesionales que desdibujan las líneas entre los diferentes géneros periodísticos.

A pesar de ello, los profesionales son conscientes de los cambios producidos y de la incorporación de nuevas prácticas. En ningún caso supone "una amenaza laboral para los periodistas en activo, sino la evolución propia de la profesión en su proceso de adaptación a las tecnologías digitales" (Sánchez y Méndez, 2013: 147).

En este sentido, Telegram ofrece opciones que podrían ser valoradas por los periodistas a la hora de utilizar la herramienta (Thorsen y Jackson 2018). Por ejemplo, el uso de chats secretos que se autodestruyen transcurrido cierto periodo de tiempo, comunicaciones encriptadas o creación de canales personalizados para el usuario. Esto provoca que los profesionales de la información vivan en un feedback constante, donde la audiencia comenta, comparte o expresa diferentes tipos de emociones sobre sus contenidos al instante.

Telegram irrumpe en las rutinas de los periodistas que trabajan en medios y empresas de comunicación estableciendo nuevas prácticas de producción y difusión periodística. Las noticias digitales producen cambios económicos, que en algunos casos borran las perspectivas laborales y la seguridad profesional de los periodistas. Sin embargo, se están impulsando nuevas formas y lenguajes periodísticos que contribuyen a una reconstrucción creativa de los valores del periodismo en la noticia (Martini, 2000). Estos residen en el interés colectivo, la inmediatez o la proximidad, así como en los principios éticos de veracidad, independencia, imparcialidad y responsabilidad. Sin duda, los desafíos digitales deben ir de la mano de la ética de la profesión (Heinonen, 2004; Micó, et al., 2008; Suárez-Villegas, 2015).

La presente investigación tiene como objetivo analizar el uso de Telegram en las rutinas periodísticas y examinar la percepción de los profesionales y estudiantes de la comunicación respecto al consumo de información. El trabajo de investigación utiliza técnicas cualitativas y cuantitativas y parte de las siguientes hipótesis: En primer lugar, que las innovaciones que presenta Telegram han permitido la penetración de la plataforma en las prácticas de los profesionales de la comunicación, principalmente, porque posibilita la comunicación interna, fidelidad con el usuario y es fuente de información personalizada, sin embargo, plantea desafíos en la profesión por su exigua implantación social. En segundo lugar, la percepción por los profesionales y estudiantes de la comunicación es que la plataforma de mensajería posibilita el consumo de información de forma inmediata y actual debido a la sencillez de uso, no así, por la participación que pueda facilitar a los usuarios.

\section{Metodología}

Para cumplir los objetivos, se ha diseñado una metodología basada en la utilización de técnicas cualitativas y cuantitativas, entre las que destacan el análisis documental (Krippendoff, 1990; Neuendorf, 2004), la entrevista y la elaboración de encuestas a profesionales y estudiantes de comunicación en España.

En primer lugar, se ha efectuado una revisión documental sobre el uso de Telegram por parte de los profesionales de la comunicación, su incursión en las rutinas periodísticas (López, et al, 2019) y su función como herramienta de distribución y consumo por parte de los profesionales (Sedano-Amundarain y Palomo-Torres, 2018).

En segundo lugar, se ha procedido a la realización de entrevistas en profundidad (Flick, 2015; Robles, 2011; Gibbs, 2012), de carácter semiestructurado, flexible y dinámico (Díaz-Bravo, Torruco-García, Martínez-Hernández y Varela-Ruiz, 2013), que aporten testimonios acerca de la utilización de Telegram en la rutina del profesional de la información, así como los posibles cambios y variaciones que ha provocado. Se ha elegido esta técnica debido a su utilidad como instrumento de 
investigación cualitativa para recabar datos (ibídem, 2013). El periodo de realización de entrevistas fue desde marzo hasta junio de 2018 y éstas se efectuaron vía telefónica.

La elección de esta muestra responde a los siguientes criterios: responsabilidad en el cargo, número de suscriptores del medio en la plataforma y originalidad de contenidos y formatos. Se ha consultado al subdirector y cofundador de Eldiario.es, la directora del equipo de redes sociales de El País, responsable de redes sociales de Verne y coordinador de Maldito Dato en Maldita.es. Para aportar una visión más técnica sobre el tema, también se ha entrevistado a un periodista y consultor en desarrollo de audiencias, negocio y producto.

Para la obtención y análisis de datos extraídos de las entrevistas, se ha empleado una matriz de variables cualitativas para tratar la información y organizar cada una de las respuestas obtenidas. Así pues, se establecieron categorías (Taylor et al, 2015) con un único principio clasificatorio que hacen alusión a una única dimensión. De este modo, se han agrupado las declaraciones en función de las siguientes variables: a) características sociodemográficas: ocupación y perfil profesional; b) hábitos de consumo de información: perfiles de acceso en Telegram, expectativas de la herramienta; c) hábitos de uso de la aplicación: valoración de uso por parte de periodistas y medios, ventajas de Telegram, tipo de información, avances respecto a otras plataformas, comparación con otras redes sociales y e) interacción con los usuarios: relación de los medios con el usuario. Posteriormente, se procedió a la comparación y triangulación (Díaz-Bravo, Torruco-García, Martínez-Hernández y Varela-Ruiz, 2013), es decir, unificación del cuerpo teórico, documentos y declaraciones para consolidar de forma significativa la interpretación. Los profesionales del periodismo entrevistados, previamente seleccionados como se ha adelantado anteriormente, han sido los siguientes (Tabla 1).

Tabla 1. Muestra de profesionales entrevistados

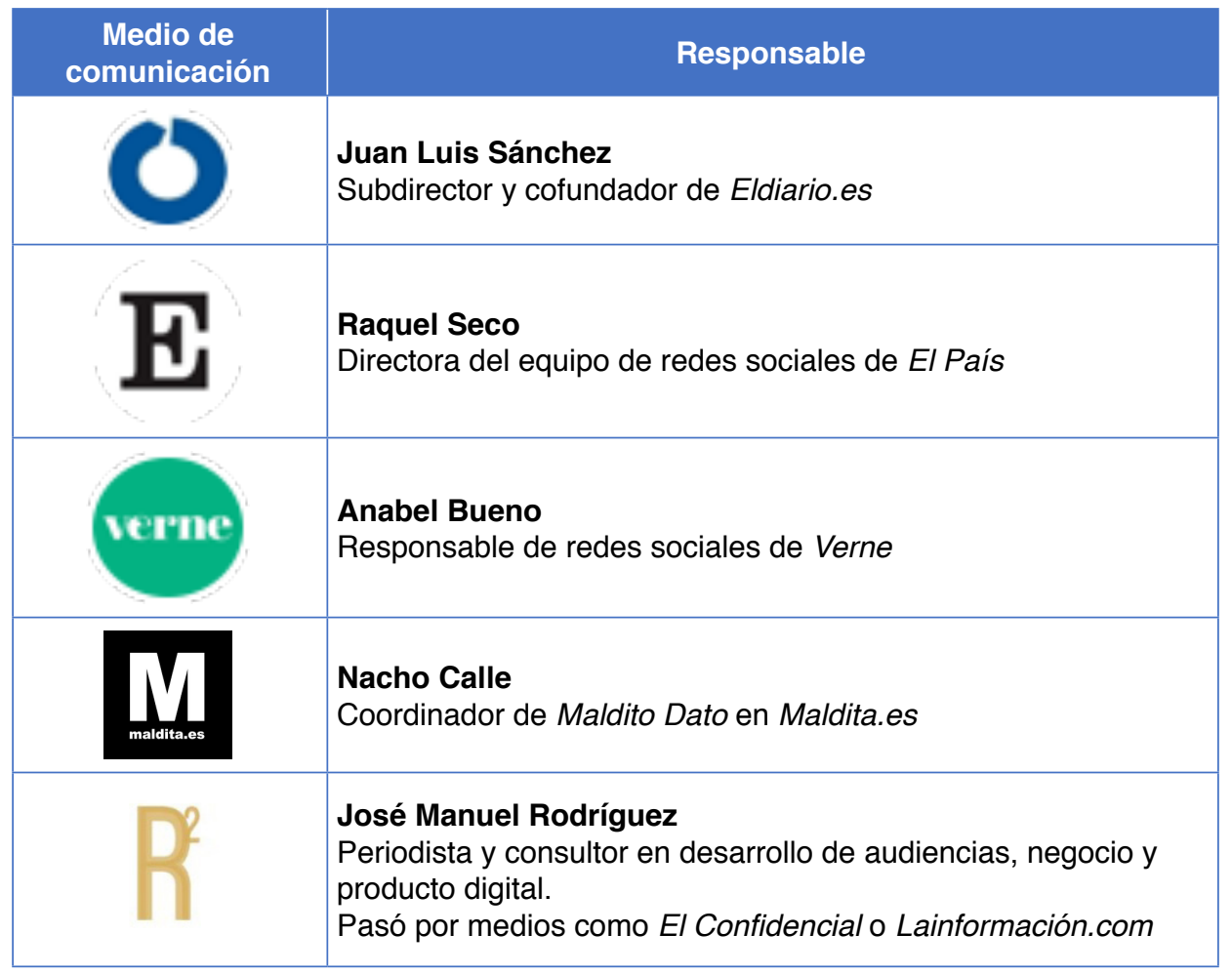

Fuente: Elaboración propia

Para determinar la percepción de los profesionales de comunicación acerca de la incursión de $T e$ legram en el ámbito periodístico, se ha procedido a la realización de encuestas (Sierra, 2008; Cea d'Ancona, 2010) a profesionales de distintos medios de comunicación, gabinetes y Asociaciones de 
Periodistas y a futuros periodistas (estudiantes de comunicación). Esta técnica ha permitido la obtención de datos objetivos (hechos) y subjetivos (opiniones, actitudes) (Igartua, 2006) para ambos casos y respecto al uso de la aplicación, consumo de información o frecuencia de acceso. Como resultado, se obtuvo un total de 321 respuestas que componen nuestra muestra (107 periodistas y 214 estudiantes o futuros profesionales). Se aplicó la técnica del muestreo no probabilístico (Scharager y Reyes, 2001; Pimienta Lastra, 2000) para su elección. El periodo de realización de encuestas ha sido desde marzo hasta diciembre de 2018.

Para las respuestas se generó una alternancia entre una variable dicotómica (sí/no) y otra politómica (elegir entre una o varias opciones). Las variables de respuesta cualitativa escritas por los participantes han sido clasificadas en categorías y subcategorías atendiendo a su contenido (canales adicionales a los que el usuario accede o las ventajas y desventajas que la audiencia encuentra en Telegram): a) ventajas de la aplicación de mensajería: interacción con usuario, recibir alertas de última hora, información amplia, formato cómodo, recibir los titulares más relevantes, posibilidad de elección, formato original, cualidades técnicas, utilidad y posibilidad de difusión de información; b) desventajas de la aplicación de mensajería: no se utiliza para informarse, no es contenido imprescindible, poco uso general, poca implantación social, no sustituye la información de los medios, preferencia por otras aplicaciones y no cumple con las expectativas; c) canales: partidos políticos y sindicatos; información especializada: deporte, política; de ocio: grupos de amigos y apuestas; E-commerce: tienda online, servicios, membresía y entretenimiento. Se han generado 33 variables dicotómicas (Sí/No) por cada categoría y que han sido comparadas en función de los factores (sexo, edad y perfil de usuario).

La combinación de ambas técnicas de investigación ha permitido alcanzar un enfoque más amplio a la hora de abordar el presente estudio. De este modo, las cifras y porcentajes alcanzados en los cuestionarios se han comparado con los resultados conseguidos en las entrevistas, con el fin de obtener una visión más global y detallada acerca del tema de investigación. Para los cuestionarios, se han utilizado los siguientes modelos (Tabla 2):

Tabla 2. Ficha técnica de la encuesta realizada a profesionales y estudiantes

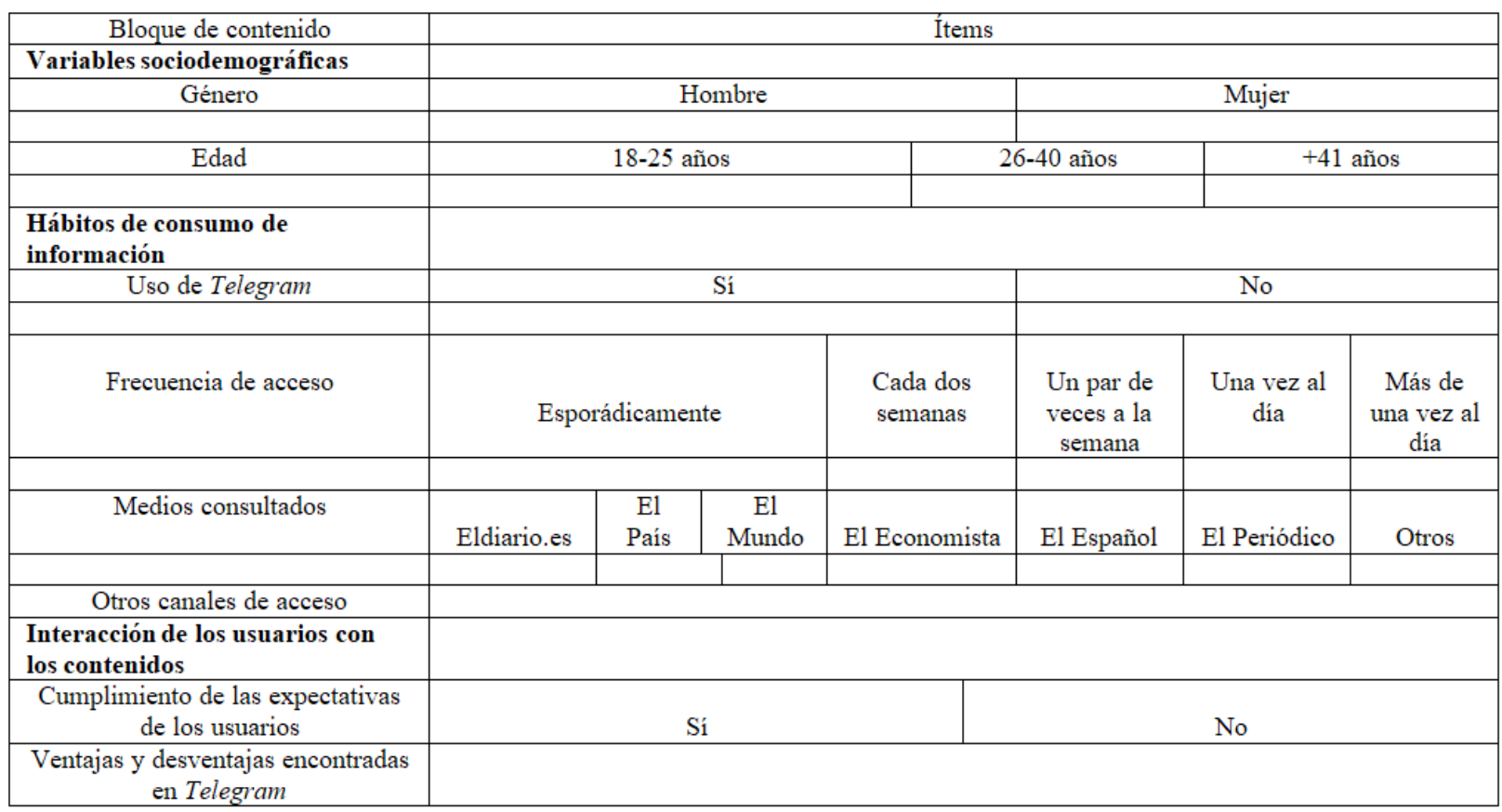

Fuente: Elaboración propia 


\section{Análisis y resultados}

De acuerdo con los datos obtenidos en la presente investigación, la acogida de Telegram como herramienta en la rutina periodística ha sido positiva por parte de los profesionales de la comunicación. Más de la mayoría de periodistas encuestados, el $80 \%(n=107)$, afirma que ha utilizado o utiliza actualmente Telegram tanto para recibir información como para difundirla, frente a un 20\% que nunca ha accedido a la aplicación ni tiene conocimiento sobre la misma.

Igualmente, más de la mitad de estudiantes encuestados $(58 \% ; n=214)$ ha utilizado la herramienta y solo el $42 \%$ nunca lo ha hecho (Gráfico 1). Es perceptible que el uso de la aplicación por parte de periodistas y estudiantes difiere. Esto se debe a la importancia que ha cobrado Telegram como herramienta rutinaria de trabajo para los profesionales de la información en las redacciones de medios. No obstante, ambos perfiles valoran como muy positivo consumir información a través de titulares más relevantes ( $27 \%$ en periodistas y $25 \%$ en estudiantes) y alertas con noticias de última hora (15\% y $11 \%$, respectivamente). Estos son dos de los aspectos más destacados en el uso de Telegram para consultar información periodística.

Gráfico 1. Uso de Telegram por parte de periodistas y estudiantes de comunicación

Periodistas

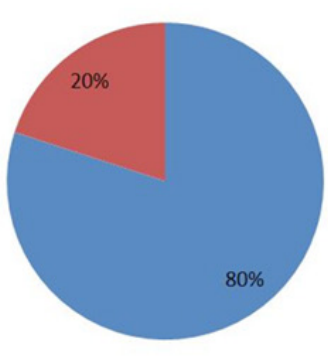

Estudiantes

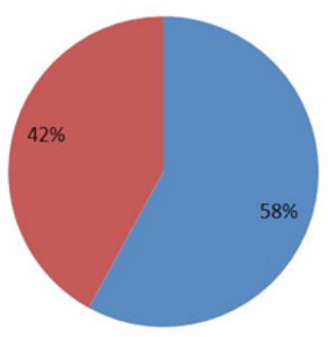

Sí lo han utilizado

No lo han

utilizado

Fuente: Elaboración propia

Este elevado porcentaje de uso de la aplicación por los profesionales de la comunicación está relacionado con las cualidades técnicas (Sánchez y Martos, 2018) que la herramienta propone. En primer lugar, Telegram ofrece la posibilidad de crear grandes grupos para distribuir contenidos, permitiendo "una cantidad de usuarios mucho mayor que en WhatsApp", como afirma Anabel Bueno (comunicación personal, 2018), responsable de redes sociales de Verne.

Esto se debe a que, mientras WhatsApp limita el número máximo de miembros a 256 integrantes, la cifra en Telegram es de 200.000 usuarios según datos del sitio web oficial de la aplicación. La posibilidad de distribución masiva se convierte en un componente útil y atractivo en la actividad del periodista. "Es tan fácil mandar un mensaje a 21.000 personas como mandar un WhatsApp a cualquier amigo" afirma Juan Luis Sánchez (2018, comunicación personal), subdirector y cofundador de Eldiario.es. Esta característica resulta también práctica para los propios medios de comunicación por la facilidad de difusión de contenidos a un público amplio. Sin embargo, al recurrir a los datos extraídos de las encuestas, se observa que tan solo un $5 \%$ de los profesionales consultados considera la distribución masiva como una ventaja de la herramienta.

Telegram ofrece sencillez de uso (Sánchez 2018, comunicación personal) y un formato cómodo, ya que la aplicación, según afirma Nacho Calle (2018, comunicación personal), coordinador de Maldito Dato en Maldita.es, "puede utilizarse en distintos soportes digitales (smartphone, tablet, ordenador...)".

Además, presenta un carácter original y novedoso, con la posibilidad de incluir todo tipo de archivos: stickers, GIFs o audios (Sánchez, 2018, comunicación personal). Los recursos multimedia se 
han convertido en una opción para distribuir información a través de los canales de Telegram de un modo más personalizado y cercano con el usuario, favoreciendo así el vínculo entre periodista y audiencia (Sánchez y Martos, 2018). En este sentido, Sánchez (2018, comunicación personal) afirma:

"Intentamos cuidarles con un tono y una relación muy directa. La idea es crear fidelidad en ese pequeño grupo que confía en nosotros tanto como para tenerle en una herramienta que sabemos que es minoritaria. Presentamos temas de interés, aunque dependiendo de la hora tienen más un tono informativo o más de entretenimiento. Intentamos dar algún guiño con GIFs o emoticonos y audios. Intentamos jugar con los formatos y con un tono diferente" (Sánchez, 2018; comunicación personal).

Las infografías (sobre encuestas electorales, estudios sociológicos...) y los vídeos informativos también cobran protagonismo en el caso de medios como El País, según afirma su directora del equipo de redes sociales, Raquel Seco (2018, comunicación personal). En cuanto a la inclusión de audios en la información, Sánchez (2018, comunicación personal) destaca este formato como uno de los más exitosos de Eldiario.es en Telegram:

"Una de las cosas que más nos ha funcionado desde el principio son pequeños audios que mandamos con el análisis de las noticias del día, cuando pasa algo especialmente relevante. Son fáciles de grabar, enviar y también de editar, si te equivocas. Porque otra de las grandes ventajas de Telegram es que te puedes equivocar al enviar algo, pero te permite flexibilidad a la hora de editar y enviar. Y eso en un medio de comunicación, que sabes que te puedes equivocar, te da seguridad y tranquilidad" (Sánchez, 2018; comunicación personal).

Gráfico 2. Ventajas de Telegram según periodistas y estudiantes

\section{Periodistas}

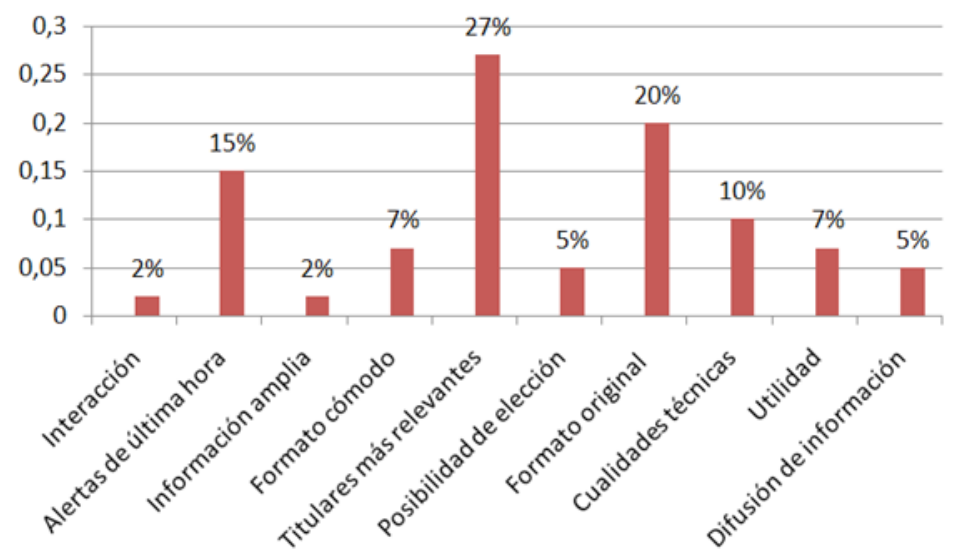

\section{Estudiantes}

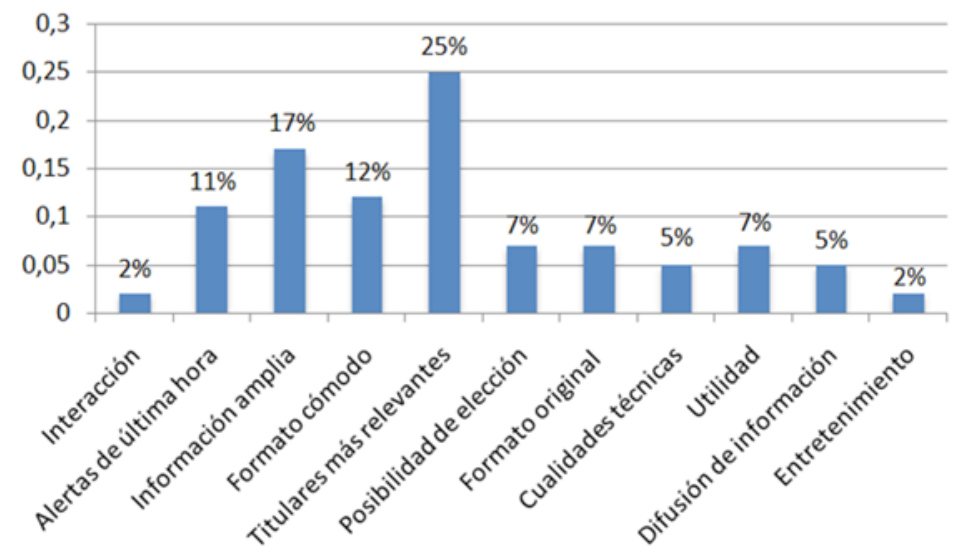

Fuente: Elaboración propia. 
Más de la mitad de los periodistas (79\%) y estudiantes (68\%) encuestados afirma que Telegram cubre sus expectativas como herramienta de difusión de contenidos, así como de consumo de información. Esto se debe principalmente a la posibilidad de recibir los titulares más relevantes o alertas de última hora y al formato original de la aplicación (Gráfico 2).

Otras de las razones de la aceptación de Telegram en la que coinciden los periodistas y estudiantes encuestados es la posibilidad de recibir avisos de última hora (15\%). La difusión de breaking news o alertas se han convertido en una práctica habitual a través de la aplicación (Seco, 2018, comunicación personal). "Telegram se usa en la redacción principalmente para últimas horas y también para distribuir contenidos que creemos que son compatibles. Pero nos centramos mucho en dar breaking news. Es lo más habitual", afirma. También destacan, aunque en menor medida, las características técnicas que la herramienta ofrece, su utilidad y la comodidad de la plataforma (Gráfico 2).

Por otro lado, se aprecia una mayor disparidad entre periodistas y estudiantes en cuanto a determinadas características técnicas. Destaca la diferencia en la percepción de que Telegram posee un formato original ( $20 \%$ en periodistas y $7 \%$ en estudiantes). Esto se debe a que los profesionales están más acostumbrados al uso de la aplicación en sus rutinas para la distribución de noticias o la comunicación en las propias redacciones. Mientras tanto, según los datos recopilados, se observa que los estudiantes se centran más en el consumo de contenidos en Telegram que en la distribución. Por ello, se aprecia una diferencia significativa entre estudiantes y periodistas en cuanto a la posibilidad de recibir información amplia a través de la aplicación (17\% en estudiantes y $2 \%$ en periodistas).

Por el contrario, el 21\% restante de periodistas encuestados afirma que Telegram no cubre sus expectativas, principalmente por el escaso uso de la herramienta para buscar información y la escasa implantación social (29\%). En el caso de los estudiantes, el 32\% manifiesta que la aplicación no cubre sus expectativas porque no la utilizan para informarse (41\%) (Gráfico 3).

Gráfico 3. Desventajas de Telegram según los periodistas y estudiantes

Periodistas

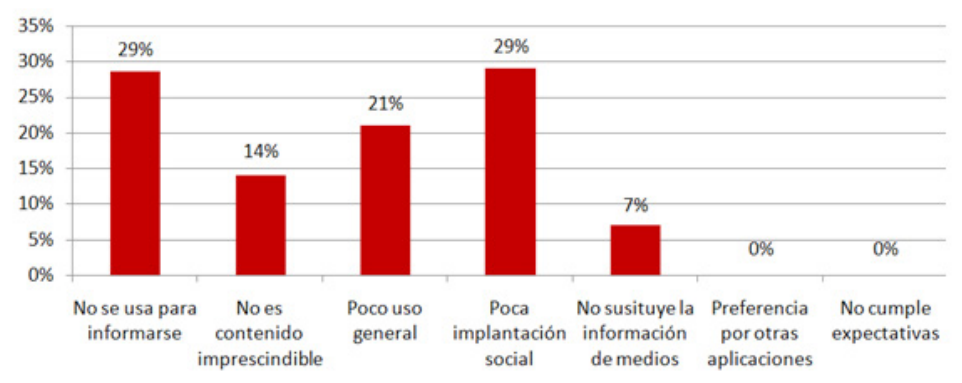

Estudiantes

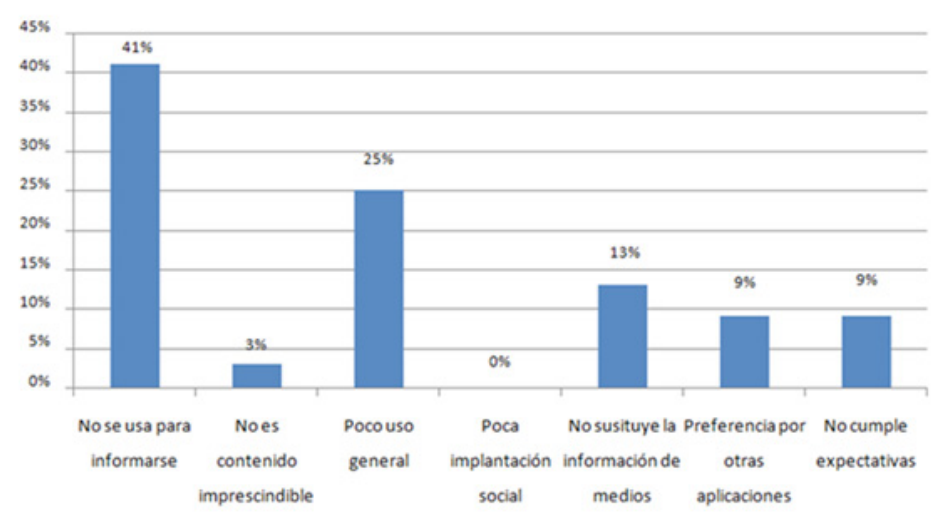

Fuente: Elaboración propia 
Se observa una amplia diferencia en cuanto a lo que ambos perfiles consideran como desventajas de Telegram. Para los periodistas, la escasa implantación de la plataforma en la sociedad (29\%) representa una importante razón para llegar a más audiencia o fidelizarla, mientras que para los estudiantes encuestados el escaso uso general de la aplicación tiene que ver con varios factores, como es la preferencia por otras aplicaciones, se informan por otras vías y porque no cumple con sus expectativas a nivel social.

Debido al hábito de trabajar con las nuevas tecnologías, la necesidad de innovar (García-Avilés, Carvajal Prieto, Arias Robles, 2018) en la profesión y la observación de las principales tendencias en las audiencias, el periodista cuenta con esta perspectiva adicional que le lleva a tener en cuenta la viabilidad o presencia social de una aplicación como un condicionante fundamental para distribuir las informaciones del medio.

Los periodistas, además del uso de Telegram como herramienta de difusión de contenidos, también afirman recurrir a la plataforma para el consumo de información. Los datos recopilados en la presente investigación muestran que el medio más consultado por los periodistas es Eldiario.es (26\%), seguido por El País (22\%). Estas preferencias coinciden con los datos de los medios más consultados en el caso de los estudiantes (Gráfico 4).

Gráfico 4. Medios de comunicación a los que acceden los periodistas y los estudiantes

\section{Periodistas}

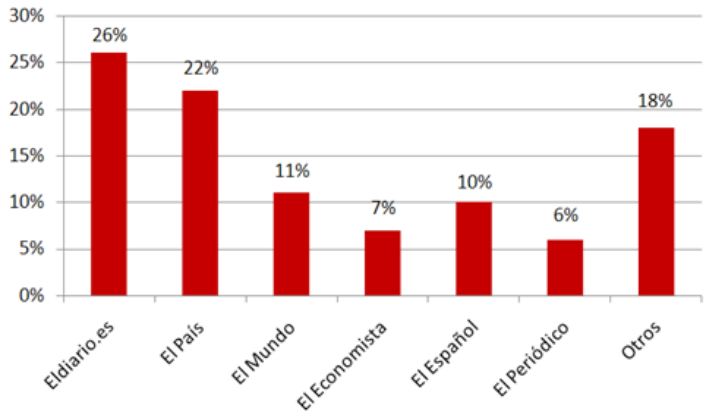

\section{Estudiantes}

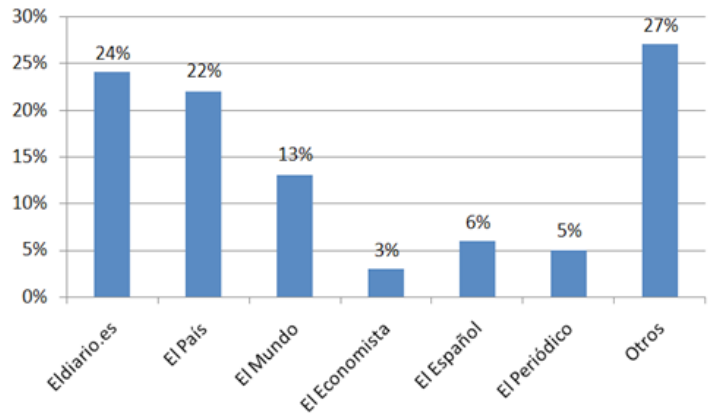

Fuente: Elaboración propia.

Además de consultar información en medios de comunicación, destaca como uso principal el acceso a Politibot ( $26 \%$ en periodistas y $11 \%$ en estudiantes), un bot (Sánchez y Sánchez, 2017) que ofrece conversaciones a tiempo real sobre las claves de la actualidad política. Los canales oficiales de partidos políticos $(17 \%)$ y canales de compra online $(9 \%)$ destacan entre las preferencias de los periodistas encuestados y los canales de apuestas $(10 \%)$ entre las de los futuros profesionales.

La posición hegemónica de Eldiario.es en Telegram se debe a que los periodistas de la redacción se han habituado al uso natural de la aplicación, tanto en las labores de difusión de información como de comunicación entre los miembros del periódico, según afirma Sánchez (2018, comunicación personal):

"Nosotros somos usuarios intensivos de Telegram, tanto de manera pública como privada. Es decir, lo utilizamos también como sistema de trabajo interno. Con lo cual, eso nos hace estar muy naturalizados con la herramienta y nos ayudó en su momento a entender cuál era el tono adecuado tan específico para un canal como éste" (Sánchez, 2018; comunicación personal).

En la misma línea, Nacho Calle (2018, comunicación personal) también destaca el importante papel de Telegram como herramienta de comunicación interna: "Lo utilizamos como medio de difusión, aunque para nosotros es más importante como plataforma de comunicación interna". 
A pesar de la evidente incursión de Telegram en la rutina de trabajo del profesional de la comunicación y las innovaciones que la herramienta presenta, su implantación todavía está en periodo de desarrollo. Un 47\% de los periodistas encuestados afirma acceder solo esporádicamente, mientras que únicamente el $20 \%$ entra en la plataforma de mensajería de forma habitual, más de una vez al día. Esta diferencia se aprecia de forma más notoria en los datos de los estudiantes de comunicación encuestados, ya que la mayoría afirma consultar Telegram esporádicamente (63\%) y tan solo un 8\% accede una o más veces al día (Gráfico 5).

Gráfico 5. Frecuencia de acceso de los periodistas y estudiantes a Telegram

\section{Periodistas}

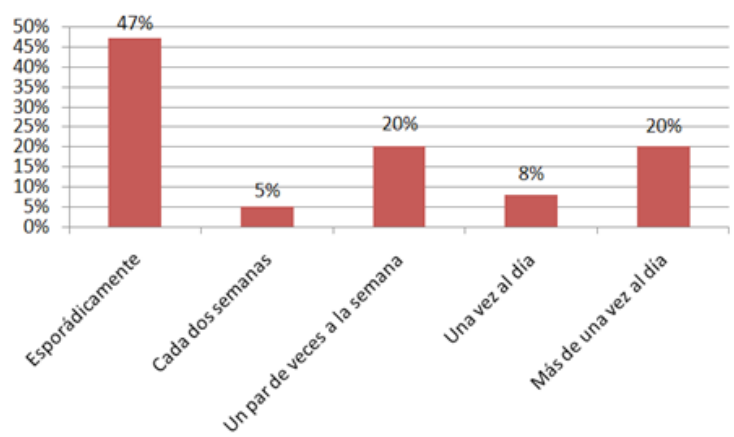

\section{Estudiantes}

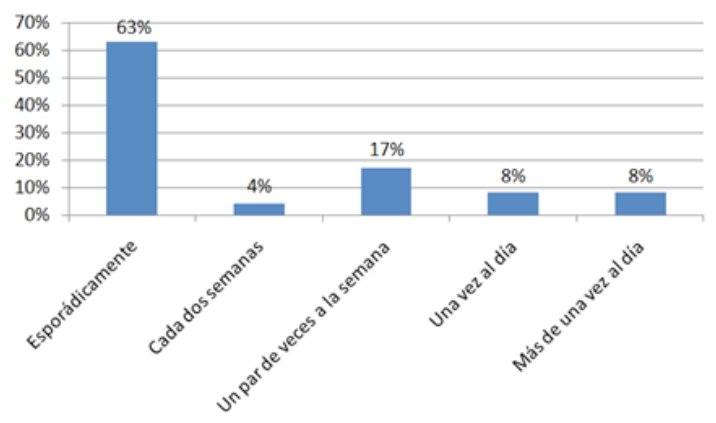

Fuente: Elaboración propia.

Telegram ofrece seguridad y privacidad (Martín Granados, 2016; Dargahi, 2017; Hintea, 2018) en sus comunicaciones, lo que resulta útil, por ejemplo, en el contacto con fuentes de información. "Si nuestro redactor que sigue a Podemos tiene que hablar con Pablo Iglesias, lo hace a través de Telegram [...] Es un canal de influencia”, afirma Sánchez (2018, comunicación personal). No obstante, la misma seguridad y encriptación ofrecida por la herramienta provoca la proliferación de fake news y la difusión de bulos a través de la aplicación de mensajería instantánea, según explica Rodríguez (2018, comunicación personal):

"Hay un componente importante a la hora de explicar la difusión de noticias falsas. La encriptación hace que las aplicaciones de mensajería no sepan lo que se está compartiendo; no son capaces de conocer los mensajes porque están encriptados. Eso da mayor seguridad y privacidad a nivel de usuario, ya que no puede ser espiado al principio" (Rodríguez, 2018; comunicación personal).

En la misma línea, desde Maldita.es se lucha contra la difusión de bulos en Telegram, según explica Calle (2018, comunicación personal):

"Hemos creado un canal de Telegram en el que vamos contando qué desmentidos vamos haciendo, de tal manera que ellos mismos, con facilidad, puedan mover en Telegram dicho desmentido [...] Y ahora, estamos desarrollando también un bot que sirve para crear un tema que desmentir" (Calle, 2018; comunicación personal).

Con el fin de evitar este tipo de prácticas, medios internacionales como BBC, Reuters, The Guardian, The New York Times o The Washington Post (Herrera-Damas, 2013) han elaborado normativas de actuación que recogen indicaciones para la regulación del comportamiento de los periodistas en estas plataformas (Sánchez, 2016). Estas directrices, denominadas social media policies (Herrera Damas, 2011), se orientan principalmente a la potenciación de prácticas como la verificación de fuentes, la rectificación en caso de error, la imparcialidad (Kovach; Rosenstiel, 2007), la integridad o el respeto, así como al rechazo de otras como la expresión de opiniones propias cuando se está informando, caer en prejuicios y favoritismos u ocultar su identidad en la red (Herrera Damas, 2013). 
Aunque desde los medios españoles analizados no se ha mencionado la existencia de estas políticas específicas para el uso de plataformas de mensajería, sí que coinciden en la importancia de las buenas prácticas y los valores éticos de la profesión. En este sentido, Rodríguez (2018, comunicación personal) añade también la encriptación como un componente importante a la hora de explicar la difusión de noticias falsas, ya que no es posible "conocer los mensajes al estar cifrados" y eso "da mayor seguridad y privacidad al usuario porque no puede ser espiado".

Mientras el periodismo evoluciona, los profesionales de la comunicación luchan por mantener el ritmo. La constante innovación y actualización de contenidos digitales en este tipo de plataformas fuerza al periodista a permanecer en una "rueda de hámster" (Breese y Luengo, 2016) de producción de noticias. Esta labor exige clics para atraer ingresos publicitarios y provoca que la identidad del periodismo derive de los códigos culturales de la esfera civil (Alexander, 2006), de las demandas de la sociedad. A su vez, esto obliga al profesional a "no pensar tanto en contenido exclusivo de Telegram, sino más bien en otro sentido, en cuáles de los contenidos con los que trabaja el medio se adaptan más a una plataforma que es móvil y social" (Seco, 2018; comunicación personal).

Como ocurre con el resto de plataformas digitales, tanto redes sociales (Facebook, Twitter) como aplicaciones de mensajería privada (WhatsApp), la influencia de Telegram también viene determinada por el factor sociológico y las variables estadísticas, como la edad. Según Rodríguez (2018, comunicación personal), "en este aspecto, la herramienta cuenta con un público más joven, con mayores facilidades para adaptarse al lenguaje tecnológico".

Se trata de un fenómeno que no solo ocurre a nivel social, sino también dentro de las propias redacciones. Según las cifras obtenidas en el presente estudio, el mayor porcentaje de periodistas que utiliza Telegram se encuentra en un intervalo de edad de entre 26 y 40 años (43\%), seguido del rango de entre 18 y 25 años (40\%). La diferencia entre rangos de edad se hace más notoria en los estudiantes de comunicación, donde el 94\% ese ubica entre 18 y 25 años (Gráfico 6). Por ello, el uso de la aplicación se vincula a una población más joven. En los profesionales, las cifras se muestran más parejas por la integración del uso de Telegram en las rutinas periodísticas. En este caso, los datos indican que las tendencias de mercado tienen mayor peso e importancia que el factor de la edad.

Gráfico 6. Diferencias en el uso de Telegram según la edad

\section{Periodistas}

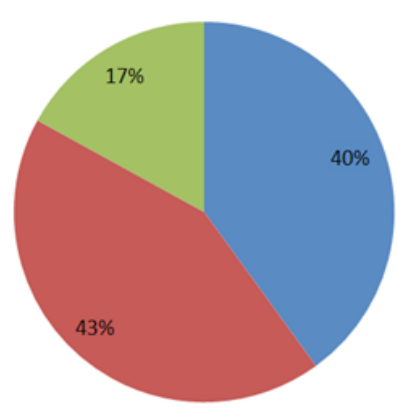

\section{Estudiantes}

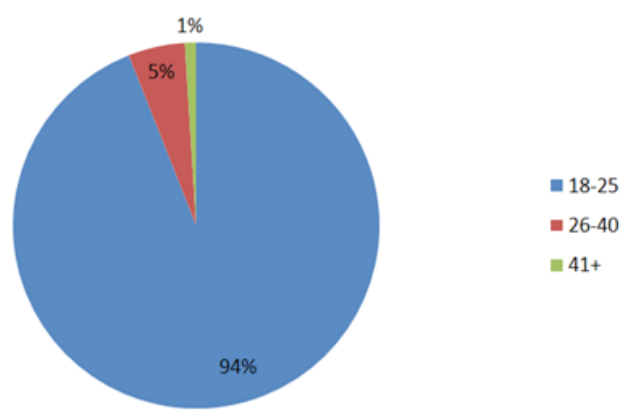

Fuente: Elaboración propia.

No obstante, los profesionales de la comunicación no son ajenos a la debilidad de Telegram en cuanto a implantación social. Su uso minoritario (Bueno, 2018; comunicación personal; Sánchez, 2018; comunicación personal) a nivel civil y la posición predominante de WhatsApp en el ámbito de las plataformas de mensajería en España (Rodríguez, 2018; comunicación personal) representan un importante inconveniente para el desarrollo de Telegram. Sus características técnicas y su utili- 
dad (Gil, 2016) han logrado que la aplicación haya irrumpido en el mercado, aunque aún presenta limitaciones de incursión social.

Actualmente, la población posee un conocimiento de WhatsApp superior al $90 \%$ y un uso promedio de la herramienta del 88\% en España (IAB Spain, 2019). Su predominio deja poco espacio a otras aplicaciones (Rodríguez, 2018; comunicación personal) y ha adquirido tal protagonismo que ha logrado introducirse en el lenguaje (Calle, 2018; comunicación personal) de la ciudadanía: "Mándame un WhatsApp", "Whatsappear", etc.

Varios de los autores consultados (Sánchez, 2018; comunicación personal; Seco, 2018; comunicación personal) hacen alusión sobre los posibles progresos que WhatsApp podría experimentar en el futuro. Si iguala o supera la oferta de Telegram en cuanto a características técnicas, esta última aplicación podría tener poco recorrido para continuar expandiéndose.

\section{Conclusiones}

La presencia de Telegram en la rutina de trabajo del periodista muestra un protagonismo creciente de la aplicación en la actividad de los medios. La mayoría de los periodistas, según el presente estudio, afirman utilizar o haber utilizado la aplicación para las tareas de distribución de contenidos y de consumo de información. Siguiendo el objetivo principal de la investigación, se observa que Telegram ha logrado postularse como una de las plataformas preferidas por los medios. Esto se debe a su utilidad y a sus cualidades técnicas para la distribución y consumo de contenidos de los propios periodistas, tanto en la búsqueda de nuevas noticias y fuentes de información, como en su papel de herramienta de comunicación interna dentro de las redacciones. De este modo, se cumple la primera hipótesis de la que parte el presente estudio, ya que estas innovaciones técnicas de Telegram han permitido su irrupción en las prácticas de los profesionales de la comunicación.

Por otra parte, la presencia de usuarios de medios de comunicación en una plataforma más minoritaria a nivel social demuestra un vínculo de compromiso entre periodistas y audiencia que se basa en valores como la actualidad, la inmediatez o la proximidad, así como la fidelidad por parte de los clientes con los contenidos ofrecidos por cada medio. La difusión de alertas de última hora o de los titulares más relevantes son algunos ejemplos de este vínculo por parte de profesionales y estudiantes de la comunicación. Los formatos como vídeos, audios y los recursos multimedia son de gran utilidad para el trato con la audiencia, ya que dotan a la comunicación de un carácter más humano, emocional, personalizado y cercano al usuario (Sánchez y Martos-Moreno, 2018). Sin duda alguna, las funciones y competencias del periodista han cambiado ante la exigencia de este nuevo desafío digital.

Sin embargo, a partir de los resultados obtenidos en el estudio, se observa que la interacción por parte de las audiencias no representa un factor importante al analizar los pros y contras de Telegram. Los periodistas y estudiantes de comunicación consultados resaltan otros aspectos relacionados con la inmediatez en el consumo de información y la sencillez de uso, más que la participación de los usuarios, tal y como se planteaba en la segunda hipótesis de partida de la investigación. Además, en el caso particular de los profesionales de la comunicación, se realiza un especial énfasis en la utilidad de la herramienta para las labores de distribución de información. En Telegram predomina un modelo de comunicación unidireccional por parte de los profesionales y los medios. Por ello, la tarea del periodista en la plataforma de mensajería se enfoca hacia la distribución de noticias de forma personalizada y dinámica, más que hacia la búsqueda de feedback o respuesta del público.

Por otra parte, Telegram también representa un arma de doble filo desde la perspectiva deontológica, especialmente por la seguridad y privacidad que ofrece. Este tipo de plataformas, a pesar de ser elementos útiles para el usuario, también provocan la circulación de bulos de información y la caída 
en el peligro de desinformar al usuario por la inmediatez. Ante el temor de que la falta de confianza de la audiencia pueda afectar la credibilidad de los medios, desde el ámbito profesional se reclama un cambio en algunas prácticas que descansan sobre la precipitación, las intromisiones y la desidia. La irrupción de Internet no altera la norma (Herrera-Damas, 2013) y, por ello, el periodista siempre ha de contrastar y verificar la información.

El asentamiento de la plataforma de mensajería en la profesión provoca un dilema entre dos corrientes definidas. Por un lado, los autores que enfocan la problemática desde la transformación digital del periodismo y la importancia de que los profesionales dispongan de competencias digitales e informáticas (López, Rodríguez y Pereira, 2017). Por otro lado, están aquellos que se acogen a la perspectiva deontológica basada en el minucioso análisis de estas nuevas plataformas, así como sus posibilidades y límites (Micó et al, 2008). En este sentido, la incorporación de herramientas como Telegram a las rutinas de los profesionales de la información aviva el debate entre la adaptación a las nuevas prácticas tecnológicas y la prevalencia de los valores éticos de la profesión.

Pero la principal desventaja de la aplicación podría residir, a la vista de nuestro estudio, en su uso minoritario y en su limitada relevancia a nivel social. Se trata de un factor fundamental a tener en cuenta para la viabilidad de los estudios científicos sobre el papel de la plataforma de mensajería en la profesión en un futuro. En la actualidad, Telegram continúa desarrollando su incursión en el periodismo y formando parte de las rutinas de medios y profesionales de la información, aún contando con el inconveniente de la supremacía de WhatsApp (Rodríguez, 2018; comunicación personal). En caso de que esta última aplicación mejore sus prestaciones técnicas (Seco, 2018; Sánchez, 2018, comunicación personal), Telegram podría tener poco margen de desarrollo. Su evolución en cuanto a implementación social será el factor que marque o no el éxito de la herramienta.

\section{Bibliografía}

Alexander, J. C., Breese, E., \& Luengo, M. (2016). The crisis of journalism reconsidered. Cambridge University Press.

Alexander, J. C. (2006). The civil sphere. En: Alexander, J.C., Breese, E., \& Luengo, M. (2016). The crisis of journalism reconsidered. Cambridge University Press.

Álvarez, J., Capelo, M. \& Álvarez, J. I. (2019). La madurez digital de la Prensa española. Estudio de caso. Revista Latina de Comunicación Social, 74, 499- 520.

Bauman, Z. (2007). Liquid modernity. Cambridge Polity Press.

Breese, E. \& Luengo, M. (2016). News innovations and enduring commitments. En: Alexander, J.C., Breese, E. \& Luengo, M. (2016). The crisis of journalism reconsidered. Cambridge University Press.

Casasús, J. M. (2001). Perspectiva ética del periodismo electrónico. Estudios Sobre El Mensaje Periodístico, 7, 49-55.

Casero, A. (2012). Beyond news papers: News consumption among Young people in the digital era. Comunicar, 20 (39), 151-158.

Cea d'Ancona, A. (2010). Métodos y técnicas de investigación cuantitativa. Editorial Síntesis.

Dargahi Nobari, A. (2017, 6 de noviembre). Analysis of Telegram: An Instant Messaging Service. CIKM 2017, Singapore.

Díaz-Bravo, L., Torruco-García, U., Martínez-Hernández, M. \& Varela-Ruiz, M. (2013). La 
entrevista, recurso flexible y dinámico. Investigación en Educación Médica, 2 (7), pp. 162-167. ISSN: 2007-865X.

Echeverría, J. (2001). Tecnociencia y sistemas de valores. En: López, J. A. \& Sánchez, J. (2001). Ciencia, Tecnología, Sociedad y Cultura en el cambio de siglo. Biblioteca Nueva.

Erdal, J. (2007). Researching Media Convergence and Crossmedia News Production. Nordicom Review, 28 (10).

Flick, U. (2015). El diseño de la investigación cualitativa. Ediciones Morata.

García, F. (2016, 02 de mayo). Políticos y jefes de prensa, en tromba a Telegram. La Vanguardia. http://www.lavanguardia.com/politica/20160502/401512151100/politicos-jefes-de-prensa-trombatelegram.html.

García-Avilés, J. A., Carvajal Prieto, M. \& Arias Robles, F. (2018). Implantación de la innovación en los cibermedios españoles: análisis de las percepciones de los periodistas. Revista Latina de Comunicación Social, 73, 369-384. DOI: 10.4185/RLCS-2018-1260

Gibbs, G. (2012). El análisis de datos cualitativos en investigación cualitativa. Ediciones Morata. Heinonen, A. (2004). Journalistic ethics in the age of net. En: Salaverría, R. \& Sádaba, C. (2004, eds). Towards new media paradigms. Content, producers, organizations and audiences, 343-356. Eunate.

Hermida, A. \& Thurman, N. (2008). A clash of cultures. The integration of user-generated content within professional journalistic frameworks at British newspaper websites. Journalismpractice, 2 (3), 343-356.

Herrera-Damas, S. (2011). Social media policies en los medios tradicionales: cuando el sentido común no es suficiente. En: Larrondo, A \& Meso, K (2011, coords.). La transformación del espacio mediático, 418-436. Universidad del País Vasco.

Herrera-Damas, S. (2013). Indicaciones recurrentes en las normativas para el uso periodístico de las redes sociales. El profesional de la información, 22 (1), 46-53.

Herrero Curiel, E. (2015). La credibilidad de las redes sociales en el ámbito periodístico. Transinformação, 27, 165-171.

Hintea, D. (2018). Forensic Analysis of the Telegram Instant Messenger Application on Android Devices. European Conference on Cyber Warfare and Security. Academic Conferences International Limited, 12, 217-223.

Igartua, J. (2006). Métodos cuantitativos de investigación en comunicación. Bosch.

Interactive Advertising Bureau Spain (2019). Estudio Anual de Redes Sociales. http://iabspain.es/ wp-content/uploads/iab_estudioredessociales_2019_vreducida.pdf.

Kaplan, A. \& Haenlein, M. (2010). Users of the world, unite! The challenges and opportunities of Social Media. Business Horizons, 53 (1), 59-68.

Kane, G. C. (2017, 04 de abril). Digital Maturity, Not Digital Transformation. MIT Sloan Review. https://sloanreview.mit.edu/article/digital-maturity-not-digital-transformation/.

Kovach, B. \& Rosenstiel, T. (2007). The elements of journalism: what news people should know and the public should expect. En: Herrera-Damas, S. (2013). Indicaciones recurrentes en las 
normativas para el uso periodístico de las redes sociales. El profesional de la información, 22 (1), 46-53.

Krippendorf, K. (1990). Metodología de análisis de contenido. Paidós.

López, X., Silva, A., Vizoso, A., Westlund, O. \& Canavilhas, J. (2019). Periodismo móvil: Revisión sistemática de la producción científica. Comunicar, 27 (59), 9-18.

López, X., Rodríguez, A. \& Pereira, X. (2017). Competencias tecnológicas y nuevos perfiles profesionales: desafíos del periodismo actual. Comunicar, 25 (53), 81-90.

Martín Granados, I. (2016, 05 de mayo). Telegram, herramienta de comunicación política. Asociación de Comunicación Política (ACOP). https://compolitica.com/telegram-herramienta-decomunicacion-politica/.

Martini, S. (2000). Periodismo, noticia y noticiabilidad. Editorial Norma.

Masip, P., Guallar, J., Suau, J., Ruiz-Caballero, C. \& Peralta, M. (2015). News and social networks: audience behavior. El profesional de la información, 24 (4), 363-370.

Meso, K., Agirreazkuenaga, I. \& Larrondo, A. (2015). Active audiences and journalism. Analysis of the quality and regulation of the user generated contents. Universidad País Vasco; Agitalpen Zerbitzua Servicio editorial.

Micó, J. L., Canavilhas, J., Masip, P. \& Ruiz, C. (2008). La ética en el ejercicio del periodismo: credibilidad y autorregulación en la era del periodismo en internet. Estudos em comunicação, 4, 15-39.

Muñoz, D. (2019, 25 de junio). Adiós a la dictadura del click: así cambiará la forma de analizar las audiencias en 2020, Medium. https://bit.ly/3h32bPB

Neuendorf, K. A. (2002). The content analysis guidebook. Thousand Oaks; Sage.

Pérez, S. \& Micó, J. (2015). El mito de la conversación global. Usos de Twitter en las redacciones periodísticas catalanas y belgas. El profesional de la información, 24 (3), 246-255.

Pimienta Lastra, R. (2000). Encuestas probabilísticas vs. no probabilísticas. Política y Cultura, 13, 263-276. http://www.redalyc.org/articulo.oa?id=26701313.

Robles, B. (2011). La entrevista en profundidad: una técnica útil dentro del campo antropofísico. Cuicuilco, 18 (52), 39-49. ISNN: 1405-7778.

Sánchez, H. M. (2018). World Wide Web. En: Ramírez, Mª \& Martínez, Á. (208, coord.). 50 Imágenes para entender la comunicación en el siglo, 370-386. Tirant Humanidades.

Sánchez, H. M. \& Méndez, S. (2013). Nuevos medios, nuevos perfiles y conectividad emocional con la audiencia: Los periodistas en la praxis profesional. CIC Cuadernos de Información y Comunicación. 18 (1), 133-149.

Sánchez, H.M. \& García, N. (2010). Las redes sociales, la información emotiva y la participación especializada. En: Quesada, M. (2010, ed.) Internet Como Fuente Generadora de Contenidos Especializados, 371-387. Universitat Pompeu Fabra.

Sánchez, H.M. (2016). Regularización de la actividad periodística y perfiles profesionales: Medios sociales y conectividad 2.0 con la audiencia. En: Sánchez, H. M. (2016, ed.) Nuevos retos para el Periodista. Innovación, creación y emprendimiento, 63-97. Tirant Humanidades. 
Sánchez, H. M. (2005). Las tecnologías y el periodismo en la televisión: desde el ayer hacia el mañana. En: Gómez y Méndez, J. M. (2005). Las tecnologías periodísticas: desde el ayer al mañana. Sociedad Española de Periodística.

Sánchez, H. M. \& Martos-Moreno, J. (2018, 25 de mayo).Telegram como nueva estrategia de comunicación e información periodística en España. XXIV Congreso Internacional de la Sociedad Española de Periodística. Post-periodismo: Entre lo real y lo virtual, Málaga, España. https://eventos.uma.es/15425/files/xxiv-congreso-internacional-de-la-sep.-post-periodismo.-entrelo-real-y-virtual.html.

Sánchez H. M. \& Sánchez M. (2017). Bots as news service and emotional connectivity with the audience. The case of Politibot. Doxa, 25, 51-68.

Scharager, J. \& Reyes, P. (2001). Muestreo no probabilístico. Escuela de Psicología; SECICO Pontificia Universidad Católica de Chile.

Sedano-Amundarain, J. \& Palomo-Torres, B. (2018). Aproximación metodológica al impacto de WhatsApp y Telegram en las redacciones. Hipertext.net, 16, 61-67.

Shu, K., Sliva, A., Wang, S., Tang, J. \& Liu, H. (2017). Fake News detection on Social Media: A data mining perspective. SIGKDD Explor Newsl, 19, 22-36.

Sierra, R. (2008). Técnicas de investigación Social. Teoría y ejercicios. Thomson.

Singer, J. (2006). The Journalist in the Network. A shifting rationale for the gatekeeping role and the objectivity norm. Tripodos, 23, 61-76.

Suárez-Villegas, J. (2015). Nuevas tecnologías y deontología periodística: comparación entre medios tradicionales y nativos digitales. El profesional de la información, 24 (4), 390-395.

Taylor, S., Bogdan, R. \& DeVault, M. (2015). Introduction to qualitative research methods. A guidebook and resource. Wiley. ISBN: 978-1-118-76721-4

Thompson, J. B. (1985). The media and modernity: a social theory of the media. En:

Masip, P., Guallar, J., Suau, J., Ruiz-Caballero, C. \& Peralta, M. (2015). News and social networks: audience behavior. El profesional de la información, 24 (4), 363-370.

Toffler, A. (1980). La tercera ola. Plaza \& Janés.

Thorsen, E. \& Jackson, D. (2018). Seven characteristics defining online news formats. Digital Journalism, 6 (7), 847-868.

Varona, D. Sánchez, M. \& Arrocha, R. (2017). Consumo de información política en dispositivos móviles en España: caracterización del usuario tipo y su interacción con las noticias. $E l$ profesional de la información, 26 (4), 641-648. 